\title{
Spatial spectroscopy for high resolution imaging
}

\author{
Arturo Villegas ${ }^{1,2, *}$ and Juan P. Torres ${ }^{1,3}$ \\ ${ }^{1}$ ICFO-Institut de Ciències Fotòniques, Mediterranean Technology Park, 08860 Castelldefels, Barcelona, Spain \\ ${ }^{2}$ The Barcelona Institute of Science and Technology, 08036 Barcelona, Spain \\ ${ }^{3}$ Department of Signal Theory and Communications, Universitat Politècnica de Catalunya, 08034 Barcelona, Spain
}

\begin{abstract}
Quantum estimation theory provides bounds for the precision in the estimation of a set of parameters that characterize a system. Two questions naturally arise: Is any of these bounds tight? And if this is the case, what type of measurements can attain such a limit? In this work we show that for phase objects, it is possible to find a tight resolution bound. Moreover one can find a set of spatial modes whose detection provides an optimal estimation of the complete set of parameters for which we propose a homodyne detection scheme. We call this method spatial spectroscopy since it mimics in the spatial domain what conventional spectroscopy methods do in the frequency domain employing many frequencies (hyperspectral imaging).
\end{abstract}

\section{Resolution limits for multi-parameter estimation of phase objects}

The quantum Cràmer-Rao inequality sets a bound for the resolution of any ensemble of unbiased estimators $\hat{\theta}=\left\{\hat{\theta}_{i}\right\}$ $(i=1 \ldots N)$ corresponding to a set of parameters $\theta=\left\{\theta_{i}\right\}$ that characterize relevant features of an object [1]. The variance of the set of unbiased estimators fulfills the matrix inequality

$$
V(\hat{\theta}) \geq F_{Q}^{-1}
$$

i.e. $\quad V(\hat{\theta})-F_{Q}^{-1}$ is a positive semi-definite matrix. $V(\hat{\theta})$ is the covariance matrix with elements $[V(\hat{\theta})]_{i j}=$ $E\left[\left(\hat{\theta}_{i}-\theta_{i}\right)\left(\hat{\theta}_{j}-\theta_{j}\right)\right]$ and $F_{Q}$ is the Quantum Fisher Information Matrix (QFIM). For a pure state $|\Psi(\theta)\rangle \equiv|\Psi\rangle[1]$, the QFIM is

$$
\left[F_{Q}\right]_{i j}=4 \operatorname{Re}\left\{\left\langle\partial_{i} \Psi \mid \partial_{j} \Psi\right\rangle-\left\langle\partial_{l} \Psi \mid \Psi\right\rangle\left\langle\Psi \mid \partial_{k} \Psi\right\rangle\right\},
$$

where $\partial_{i}$ corresponds to the partial derivative with respect to the $i$-th parameter. In general, Eq. (1) is not a tight precision bound, so $F_{Q}^{-1}$ is not always the most informative bound. However if

$$
\operatorname{Im}\left\{\left\langle\partial_{i} \Psi \mid \partial_{j} \Psi\right\rangle\right\}=0, \quad \forall i, j \in[1, N]
$$

the inequality in Eq. (1) is tight and it is attainable when using the appropriate (optimal) measurement [2, 3].

Quantum estimation theory is of great relevance for optical probing and imaging. It has raised great interest in the last few years as a tool to determine the ultimate resolution limits. For instance, contrary to common belief, it has been demonstrated that one can distinguish the location of two incoherent point sources no matter how close they are to each other [4-7].

*e-mail: Arturo.Villegas@icfo.eu
More general problems can be analyzed. Consider the probing of a phase object. The illumination beam can be modelled as $M$ independent photons whose quantum state is pure. The state of each illumination photon is

$$
|\Psi\rangle=\int \mathrm{d} \mathbf{x} \Psi_{0}(\mathbf{x}) a^{\dagger}(\mathbf{x})|\mathrm{vac}\rangle,
$$

where $\mathbf{x}$ is the spatial coordinate, $a^{\dagger}(\mathbf{x})$ is the electromagnetic creation operator, $\Psi_{0}(\mathbf{x})$ is the spatial profile of the illumination beam and $|\mathrm{vac}\rangle$ is the electromagnetic vacuum state. After the interaction with the phase object, the quantum state of a reflected (or transmitted) photon is

$$
|\Psi(\theta)\rangle=\int \mathrm{d} \mathbf{x} \Psi(\mathbf{x}, \theta) a^{\dagger}(\mathbf{x})|\mathrm{vac}\rangle
$$

where $\Psi(\mathbf{x}, \theta)=\Psi_{0}(\mathbf{x}) \exp [i \varphi(\mathbf{x}, \theta)]$. Here $\varphi(\mathbf{x}, \theta)$ is the phase imprinted on the field by the object under study.

The quantum state in Eq. (5) fulfills the condition (3) that allows to determine the best resolution that can be attained when estimating the parameters of the object. This is of great interest since one can a priori determine the limits of what can be measured in different scenarios. Thus, the corresponding resolution bound depends on the type of interaction between light and object and on the quantum nature of the illumination.

\section{Spatial mode projection as imaging method}

If the condition given by Eq. (3) is fulfilled, it has been theoretically demonstrated that projecting light after interacting with the object onto a set quantum states $\left\{\left|\gamma_{i}\right\rangle\right\}$ formed by a particular set of spatial modes should allow to achieve the fundamental resolution limit given by Eq. (1) 
[3]. The quantum states corresponding to these modes are combinations of the quantum states $\left|\partial_{i} \Psi\right\rangle$.

How to measure such projections? In a series of pioneering experiments, Delaubert et al. [8, 9] considered a homodyne detection scheme to measure with great resolution two parameters of a Gaussian beam: 1) the displacement and 2) the tilt; both with respect to a reference axis. For the spatial shape of the mode in the local oscillator they chose the one resulting from deriving a Gaussian mode, the $\mathrm{TEM}_{10}$. In their experiments there are two particular aspects that are not obvious to hold in a general imaging scheme for phase objects: i) the spatial mode carrying information about the displacement and tilt is the same, i.e., $\left|\partial_{1} \Psi\right\rangle \propto\left|\partial_{2} \Psi\right\rangle$ and ii) it is possible to discriminate the values of the displacement and tilt by changing the phase of the local oscillator.

In this work we show how a homodyne detection scheme allows the estimation of $N$ parameters that characterize an arbitrary phase object when using a particular set of spatial modes in the local oscillator. We thus generalize the method put forward by Delaubert et al. and show that this method is generally useful for measuring phase objects.

For the sake of clarity we present the case of the estimation of two parameters, $\theta_{1}$ and $\theta_{2}$. We choose the reference state $\left|\Psi_{0}\right\rangle \equiv\left|\Psi\left(\theta_{0}\right)\right\rangle$ for fixed values of the parameters $\theta_{0}=\left(\theta_{1_{0}}, \theta_{2_{0}}\right)$. Using $\Delta_{1}=\theta_{1}-\theta_{1_{0}}$ and $\Delta_{2}=\theta_{2}-\theta_{2_{0}}$, the state that describes photons after reflection (transmission) can be written as:

$$
\left|\Psi\left(\theta_{1}, \theta_{2}\right)\right\rangle=\Gamma_{0}\left|\Psi_{0}\right\rangle+\Gamma_{1}\left|\gamma_{1}\right\rangle+\Gamma_{2}\left|\gamma_{2}\right\rangle .
$$

$\left|\Psi_{0}\right\rangle,\left|\gamma_{1}\right\rangle$ and $\left|\gamma_{2}\right\rangle$ form an orthonormal basis of vectors. The coefficients in expression (6) are explicitly: $\Gamma_{0}=1+\Delta_{1} A+\Delta_{2} B, \Gamma_{1}=\Delta_{1} N_{1}+\Delta_{2}\left(C-A^{*} B\right) / N_{1}$ and $\Gamma_{2}=\Delta_{2} N_{2}$. $A, B$ and $C$ are complex numbers corresponding to the mode overlaps $A=\left\langle\Psi_{0} \mid \partial_{1} \Psi\right\rangle, B=\left\langle\Psi_{0} \mid \partial_{2} \Psi\right\rangle$ and $C=\left\langle\partial_{1} \Psi \mid \partial_{2} \Psi\right\rangle . \quad N_{1}$ and $N_{2}$ are normalization constants ensuring $\left\langle\gamma_{i} \mid \gamma_{j}\right\rangle=\delta_{i j}$. All constants are determined by the object and parameters under consideration.

Writting explicitly the coefficients $\Gamma_{i}$ in terms of the real and imaginary parts of $A, B$ and $C$, equation (6) shows that by projecting onto the spatial mode $\left|\gamma_{2}\right\rangle$ one can determine $\Delta_{2}$ and equivalently, by projecting onto the spatial mode $\left|\gamma_{1}\right\rangle$ and setting the phase difference between probe and the local oscillator to $0\left(\operatorname{Re}\left\{\Gamma_{1}\right\}\right)$ one can determine $\Delta_{1}$. Alternatively, it is sufficient to project onto the spatial mode $\left|\gamma_{1}\right\rangle$. When the phase difference is $\pi / 2\left(\operatorname{Im}\left\{\Gamma_{1}\right\}\right)$ one can determine $\Delta_{2}$; then setting the phase difference to 0 allows to determine $\Delta_{1}$.

This procedure is general and applies to any phase object characterized by two parameters; an example is hereby presented. Consider the estimation of the sidewall angle $\beta \equiv \theta_{1}$ and height $h \equiv \theta_{2}$ of a cliff-like nanostructure. The transverse section is modelled as $S(x)=\frac{h}{2}(1+\tanh \alpha x)$, with $\alpha=2 \tan \beta / h$. The input beam is assumed to have a Gaussian profile $\Psi_{0}(x)$ of width $\sigma=\lambda / \pi N A, \lambda$ is the wavelength of the probe beam and $N A$ the numerical aperture of the imaging system. The phase added to the field after interaction with the cliff-like structure is $\varphi(x, \theta)=k h(1-\tanh \alpha x)$. Using a homodyne detection scheme with the mode $\left|\gamma_{1}\right\rangle$ in the local oscillator one can measure the coefficient $\Gamma_{1}$ in Eq. (6) and determine the parameters $\beta$ and $h$ by setting the phase difference to 0 and $\pi / 2$, respectively.

\section{Spatial spectroscopy as an imaging technique}

Spectroscopy is the study of the interaction between matter and electromagnetic radiation. In the most usual case, spectroscopic schemes choose one or more wavelengths to interrogate a sample and obtain information about particular aspects of interest, such as the presence or concentration of a chemical compound. Here we choose spatial modes to obtain information about the spatial properties of the object. This is why we term the technique spatial spectroscopy, the use of one or more spatial modes to obtain information of particular spatial features of the object.

The proposed scheme also allows to determine the modal decomposition (amplitude and phase) of the light reflected (or transmitted) from the object in a convenient basis. Although this does not always correspond to an optimal measurement (the conditions for such measurement to be optimal will be shown), it might be an useful / alternative imaging technique as one can select spatial modes that are more sensitive to the interaction with the object. When the basis are modes embedded with orbital angular momentum, this is referred as spiral imaging [10].

\section{References}

[1] C.W. Helstrom, Journal of Statistical Physics 1, 231 (1969)

[2] K. Matsumoto, in Asymptotic Theory Of Quantum Statistical Inference: Selected Papers (2005), pp. 305-350

[3] L. Pezzè, M.A. Ciampini, N. Spagnolo, P.C. Humphreys, A. Datta, I.A. Walmsley, M. Barbieri, F. Sciarrino, A. Smerzi, Physical review letters 119, 130504 (2017)

[4] M. Tsang, Optica 2, 646 (2015)

[5] M. Tsang, R. Nair, X.M. Lu, Physical Review X 6, 031033 (2016)

[6] M. Paúr, B. Stoklasa, Z. Hradil, L.L. Sánchez-Soto, J. Rehacek, Optica 3, 1144 (2016)

[7] J. Řehaček, Z. Hradil, B. Stoklasa, M. Paúr, J. Grover, A. Krzic, L. Sánchez-Soto, Physical Review A 96, 062107 (2017)

[8] V. Delaubert, N. Treps, M. Lassen, C.C. Harb, C. Fabre, P.K. Lam, H.A. Bachor, Physical Review A 74, 053823 (2006)

[9] V. Delaubert, N. Treps, C. Fabre, H.A. Bachor, P. Réfrégier, EPL (Europhysics Letters) 81, 44001 (2008)

[10] L. Torner, J.P. Torres, S. Carrasco, Optics express 13, 873 (2005) 